ANNALES

POLONICI MATHEMATICI

$90.3(2007)$

\title{
On the convergence of sequences of iterates of random-valued vector functions
}

\author{
by RaFAŁ KapicA (Katowice)
}

\begin{abstract}
Given a probability space $(\Omega, \mathcal{A}, P)$ and a subset $X$ of a normed space we consider functions $f: X \times \Omega \rightarrow X$ and investigate the speed of convergence of the sequence $\left(f^{n}(x, \cdot)\right)$ of the iterates $f^{n}: X \times \Omega^{\mathbb{N}} \rightarrow X$ defined by $f^{1}(x, \omega)=f\left(x, \omega_{1}\right)$, $f^{n+1}(x, \omega)=f\left(f^{n}(x, \omega), \omega_{n+1}\right)$.
\end{abstract}

Assume that $(\Omega, \mathcal{A}, P)$ is a probability space and $X$ is a nonempty subset of a separable normed space. Let $\mathcal{B}$ stand for the $\sigma$-algebra of all Borel subsets of $X$. We say that $f: X \times \Omega \rightarrow X$ is a random-valued vector function if it is measurable with respect to the product $\sigma$-algebra $\mathcal{B} \otimes \mathcal{A}$. We define the iterates $f^{n}, n \in \mathbb{N}$, on the set $X \times \Omega^{\infty}$ as follows:

$$
\begin{gathered}
f^{1}\left(x, \omega_{1}, \omega_{2}, \ldots\right)=f\left(x, \omega_{1}\right), \\
f^{n+1}\left(x, \omega_{1}, \omega_{2}, \ldots\right)=f\left(f^{n}\left(x, \omega_{1}, \omega_{2}, \ldots\right), \omega_{n+1}\right) .
\end{gathered}
$$

These iterates were defined independently in [4] and [5] and then studied also in [10], [2] in the scalar case and in [7] in the vector case. They are useful for instance in solving functional-integral equations (see, e.g., [3], [6]). In [7] some conditions are established which guarantee the convergence (a.s. and in $\left.L^{1}\right)$ of $\left(f^{n}(x, \cdot)\right)$ with respect to the product measure.

For a real function $f$ of the form

$$
f(x)=\Phi x+x^{1+\alpha} G(x)
$$

where $\alpha$ is a positive number, $G$ is a bounded function and $\Phi \in[0,1]$, we have very useful theorems of W. J. Thron [12] which say how fast the sequence of iterates converges to zero, the unique fixed point, depending on whether $\Phi \in(0,1), \Phi=0$ or $\Phi=1$ (see also [11; $\S 1.3]$ ). Some stochastic versions of Thron's theorems were obtained in [2] for scalar functions and

2000 Mathematics Subject Classification: Primary 39B12; Secondary 37H99, 60F15.

Key words and phrases: random-valued vector functions, sequences of iterates, speed of convergence. 
in [8] for vector functions with derivative satisfying the assumptions of the Krein-Rutman theorem [9] (cf. also [13], [14]).

The present paper is intended as an attempt to get results on the speed of convergence of iterates of a random-valued vector function $f: X \times \Omega \rightarrow X$ of the form

$$
f(x, \omega)=\Phi(\omega) x+H(x) G(x, \omega)
$$

in the case where $X$ is a subset of a separable normed space.

Let $f: X \times \Omega \rightarrow X$ be a random-valued function on $(\Omega, \mathcal{A}, P)$. Then $f^{n}: X \times \Omega^{\infty} \rightarrow X$ is a random-valued function on the product probability space $\left(\Omega^{\infty}, \mathcal{A}^{\infty}, P^{\infty}\right)$. More exactly, the $n$th iterate $f^{n}$ is $\mathcal{B} \otimes \mathcal{A}_{n}$-measurable, where $\mathcal{A}_{n}$ denotes the $\sigma$-algebra of all sets of the form

$$
\left\{\left(\omega_{1}, \omega_{2}, \ldots\right) \in \Omega^{\infty}:\left(\omega_{1}, \ldots, \omega_{n}\right) \in A\right\}
$$

with $A$ from the product $\sigma$-algebra $\mathcal{A}^{n}$.

We start with the simplest case where $f$ has the form

$$
f(x, \omega)=x \Phi(\omega) \quad \text { for } x \in X \text { and } \omega \in \Omega,
$$

improving in particular Proposition 2 of [7] where the $L^{1}$-boundedness of $\left(f^{n}(x, \cdot)\right)$ was additionally assumed.

Proposition 1. Assume that $\Omega$ is not an atom of $P, x \in X \backslash\{0\}$ and $f$ has the form (1) with $\Phi: \Omega \rightarrow \mathbb{R}$ integrable. Then the sequence $\left(f^{n}(x, \cdot)\right)$ is uniformly integrable if and only if either $E|\Phi|<1$ or $|\Phi|=1$ a.s.

Proof. Clearly

$$
f^{n}(x, \omega)=x \prod_{k=1}^{n} \Phi\left(\omega_{k}\right) \quad \text { on } \Omega^{\infty} .
$$

Assume that the sequence $\left(f^{n}(x, \cdot)\right)$ is uniformly integrable. We shall show that

$$
E|\Phi| \leq 1
$$

Suppose not and let $A$ be a measurable set such that

$$
P(A)<1 \text { and } \int_{A}|\Phi| d P>0 .
$$

On account of (2) for all $m, n \in \mathbb{N}$ we have

$$
\int_{\left\{\omega \in \Omega^{\infty}: \omega_{1}, \ldots, \omega_{m} \in A\right\}}\left\|f^{m+n}(x, \cdot)\right\| d P^{\infty}=\|x\|\left(\int_{A}|\Phi| d P\right)^{m}(E|\Phi|)^{n} .
$$

Hence

$$
\sup _{n \in \mathbb{N}} \int_{\left\{\omega \in \Omega^{\infty}: \omega_{1}, \ldots, \omega_{m} \in A\right\}}\left\|f^{n}(x, \cdot)\right\| d P^{\infty}=\infty \quad \text { for } m \in \mathbb{N} .
$$


This together with

$$
\lim _{m \rightarrow \infty} P^{\infty}\left(\left\{\omega \in \Omega^{\infty}: \omega_{1}, \ldots, \omega_{m} \in A\right\}\right)=\lim _{m \rightarrow \infty} P(A)^{m}=0
$$

contradicts the uniform integrability of $\left(f^{n}(x, \cdot)\right)$ and ends the proof of (3). From (2) and (3) it follows that $\left(f^{n}(x, \cdot)\right)$ is $L^{1}$-bounded. Due to [7; Proposition 2] we have $E|\Phi|<1$ or $|\Phi|=1$ a.s. as desired.

According to (2) we have

$$
E\left\|f^{n}(x, \cdot)\right\|=\|x\|(E|\Phi|)^{n} \quad \text { for every } n \in \mathbb{N},
$$

and if $E|\Phi|<1$, then the sequence $\left(f^{n}(x, \cdot)\right)$ converges to 0 in $L^{1}$; in particular it is uniformly integrable. In the case where $|\Phi|=1$ a.s., the uniform integrability of $\left(f^{n}(x, \cdot)\right)$ is obvious.

Clearly, if $\Omega$ is an atom of $P$, then every sequence of integrable random variables is uniformly integrable. In view of Proposition 1 we may conclude that if $\Omega$ is not an atom of $P, f$ has the form (1) and $x \in X \backslash\{0\}$, then $\left(f^{n}(x, \cdot)\right)$ converges in $L^{1}$ to zero if and only if $E|\Phi|<1$. Moreover, if $-\infty<E \log |\Phi|<0$, then it also converges almost surely, which follows from

$$
\left|\prod_{k=1}^{n} \Phi\left(\omega_{k}\right)\right|=\left(\exp \left\{\frac{1}{n} \sum_{k=1}^{n} \log \left|\Phi\left(\omega_{k}\right)\right|\right\}\right)^{n}
$$

and from the Kolmogorov strong law of large numbers. Obviously, the speed of convergence of (2) depends on the real factor $\prod_{k=1}^{n} \Phi\left(\omega_{k}\right)$ only.

We shall show that the above fact holds in a more general setting, when the random-valued function has a more general form and instead of one function we "iterate" a sequence of random-valued functions $\varphi_{n}: X \times \Omega_{n} \rightarrow X$, where $\Omega_{n}$ is an arbitrary probability space. In this case by the $n$th iterate we understand the random-valued function $f^{n}: X \times \prod_{k=1}^{\infty} \Omega_{k} \rightarrow X$ where

$$
f^{0}\left(x, \omega_{1}, \omega_{2}, \ldots\right)=x, \quad f^{n}\left(x, \omega_{1}, \omega_{2}, \ldots\right)=\varphi_{n}\left(f^{n-1}\left(x, \omega_{1}, \omega_{2}, \ldots\right), \omega_{n}\right)
$$

(cf. [8; Remark 3.1]).

Suppose now that $\varphi_{n}: X \times \Omega_{n} \rightarrow X$ has the form

$$
\varphi_{n}(x, \omega)=\Phi_{n}(\omega) x+H_{n}(x) G_{n}(x, \omega),
$$

where $\Phi_{n}: \Omega_{n} \rightarrow(0, \infty)$ is a positive random variable, $H_{n}: X \rightarrow \mathbb{R}$ is Borel and $G_{n}: X \times \Omega_{n} \rightarrow X$ is a random-valued function for every $n \in \mathbb{N}$. Clearly $\varphi_{n}$ and their iterates $f^{n}$ are random-valued functions. Following [2] we consider the following two conditions:

(i) for every $n \in \mathbb{N}$ there exists a nonnegative random variable $M_{n}$ and positive constants $\alpha, \beta_{n}$ such that

$$
\begin{gathered}
\left\|G_{n}(x, \omega)\right\| \leq M_{n}(\omega) \quad \text { for } x \in X, \omega \in \Omega_{n}, \\
E\left(\left\|\varphi_{n}(x, \cdot)\right\|^{\alpha}\right) \leq \beta_{n}\|x\|^{\alpha} \quad \text { for } x \in X
\end{gathered}
$$


and

$$
\limsup _{n \rightarrow \infty}\left(\beta_{1} \cdots \beta_{n} E\left(M_{n} / \Phi_{n}\right)\right)^{1 / n}<1
$$

(ii) $x^{*}$ is a continuous linear functional such that

$$
x^{*} x>0, \quad\left|H_{n}(x)\right| \leq x^{*} x\|x\|^{\alpha} \quad \text { for } x \in X, n \in \mathbb{N} .
$$

In what follows we denote by $\Omega^{\infty}$ the product $\prod_{n=1}^{\infty} \Omega_{n}$ equipped with the product measure. We start with the following result.

THEOREM 1. If (i) holds, then for all $x \in X$ and almost every $\omega \in \Omega^{\infty}$ the sequence

$$
\left(x^{*} \frac{f^{n}(x, \omega)}{\prod_{k=1}^{n} \Phi_{k}\left(\omega_{k}\right)}\right)
$$

converges to a positive random variable for every $x^{*}$ satisfying (ii).

This theorem will be proved together with the following one.

Theorem 2. Assume (i) and (ii) hold for an $x^{*}$. Then, for all $x \in X$,

$$
0<\liminf _{n \rightarrow \infty} \frac{\left\|f^{n}(x, \omega)\right\|}{\prod_{k=1}^{n} \Phi_{k}\left(\omega_{k}\right)} \quad \text { and } \quad \limsup _{n \rightarrow \infty} \frac{\left\|f^{n}(x, \omega)\right\|}{\prod_{k=1}^{n} \Phi_{k}\left(\omega_{k}\right)}<\infty \quad \text { a.s. }
$$

Moreover, if additionally the sequence $\left(\log \Phi_{n}\left(\omega_{n}\right)\right)$ satisfies the Kolmogorov strong law of large numbers, then, for $x \in X$,

$$
\lim _{n \rightarrow \infty}\left(\log \sqrt[n]{\left\|f^{n}(x, \cdot)\right\|}-\frac{1}{n} \sum_{k=1}^{n} E \log \Phi_{k}\right)=0 \quad \text { a.s. }
$$

In particular, if the $\Phi_{n}$ are identically distributed and $E\left|\log \Phi_{1}\right|<\infty$, then

$$
\lim _{n \rightarrow \infty} \sqrt[n]{\left\|f^{n}(x, \cdot)\right\|}=\exp \left\{E \log \Phi_{1}\right\} \quad \text { a.s. }
$$

Proofs. Let $x^{*}$ satisfy (ii). Since

$$
0<\frac{x^{*} f^{n}(x, \omega)}{x^{*} f^{n-1}(x, \omega)}=\Phi_{n}\left(\omega_{n}\right)+\frac{H_{n}\left(f^{n-1}(x, \omega)\right)}{x^{*} f^{n-1}(x, \omega)} x^{*} G_{n}\left(f^{n-1}(x, \omega), \omega_{n}\right),
$$

we have

$$
x^{*} f^{n}(x, \omega)=x^{*} x \prod_{k=1}^{n}\left(\Phi_{k}\left(\omega_{k}\right)+\frac{H_{k}\left(f^{k-1}(x, \omega)\right)}{x^{*} f^{k-1}(x, \omega)} x^{*} G_{k}\left(f^{k-1}(x, \omega), \omega_{k}\right)\right) .
$$

Cosequently,

$$
x^{*} \frac{f^{n}(x, \omega)}{\prod_{k=1}^{n} \Phi_{k}\left(\omega_{k}\right)}=x^{*} x \prod_{k=1}^{n}\left(1+\frac{H_{k}\left(f^{k-1}(x, \omega)\right)}{x^{*} f^{k-1}(x, \omega)} \frac{x^{*} G_{k}\left(f^{k-1}(x, \omega), \omega_{k}\right)}{\Phi_{k}\left(\omega_{k}\right)}\right) .
$$


To get the convergence of (5) to a positive and finite limit it is enough to show that the series

$$
\sum_{n=1}^{\infty}\left|\frac{H_{n}\left(f^{n-1}(x, \omega)\right)}{x^{*} f^{n-1}(x, \omega)} \frac{x^{*} G_{n}\left(f^{n-1}(x, \omega), \omega_{n}\right)}{\Phi_{n}\left(\omega_{n}\right)}\right|
$$

converges. To do this observe that by (i) and (ii) we have

$$
\left|\frac{H_{n}\left(f^{n-1}(x, \omega)\right)}{x^{*} f^{n-1}(x, \omega)} \frac{x^{*} G_{n}\left(f^{n-1}(x, \omega), \omega_{n}\right)}{\Phi_{n}\left(\omega_{n}\right)}\right| \leq\left\|x^{*}\right\|\left\|f^{n-1}(x, \omega)\right\|^{\alpha} \frac{M_{n}\left(\omega_{n}\right)}{\Phi_{n}\left(\omega_{n}\right)}
$$

and

$$
E\left(\left\|f^{n}(x, \cdot)\right\|^{\alpha}\right) \leq \beta_{n} E\left(\left\|f^{n-1}(x, \cdot)\right\|\right)^{\alpha} \leq \cdots \leq \beta_{n} \cdots \beta_{1}\|x\|^{\alpha},
$$

whence according to (4) the series

$$
\sum_{n=1}^{\infty} E\left(\left\|f^{n-1}(x, \cdot)\right\|^{\alpha}\right) E\left(M_{n} / \Phi_{n}\right)
$$

converges. Consequently, for every $x \in X$ and a.s. $\omega \in \Omega^{\infty}$ the series (7) converges for every $x^{*}$ satisfying (ii). This ends the proof of Theorem 1 and of the first part of (6).

Fix now $x \in X$. Then

$$
\begin{aligned}
\frac{\left\|f^{n}(x, \omega)\right\|}{\left\|f^{n-1}(x, \omega)\right\|} & \leq \Phi_{n}\left(\omega_{n}\right)+\frac{\left|H_{n}\left(f^{n-1}(x, \omega)\right)\right|\left\|G_{n}\left(f^{n-1}(x, \omega), \omega_{n}\right)\right\|}{\left\|f^{n-1}(x, \omega)\right\|} \\
& \leq \Phi_{n}\left(\omega_{n}\right)+\left\|x^{*}\right\|\left\|f^{n-1}(x, \omega)\right\|^{\alpha} M_{n}\left(\omega_{n}\right),
\end{aligned}
$$

whence

$$
\frac{\left\|f^{n}(x, \omega)\right\|}{\prod_{k=1}^{n} \Phi_{k}\left(\omega_{k}\right)} \leq\|x\| \prod_{k=1}^{n}\left(1+\left\|x^{*}\right\|\left\|f^{k-1}(x, \omega)\right\|^{\alpha} \frac{M_{k}\left(\omega_{k}\right)}{\Phi_{k}\left(\omega_{k}\right)}\right) .
$$

Since the convergence of the series (8) implies a.s. convergence of the product on the right hand side, this inequality proves the second part of (6).

The second assertion of Theorem 2 follows easily from (6) and from the equality

$$
\begin{aligned}
\log \sqrt[n]{\left\|f^{n}(x, \omega)\right\|} & -\frac{1}{n} \sum_{k=1}^{n} E \log \Phi_{k} \\
= & \frac{1}{n} \log \frac{\left\|f^{n}(x, \omega)\right\|}{\prod_{k=1}^{n} \Phi_{k}\left(\omega_{k}\right)}+\frac{1}{n} \sum_{k=1}^{n}\left(\log \Phi_{k}\left(\omega_{k}\right)-E \log \Phi_{k}\right) .
\end{aligned}
$$

We now deduce a corollary concerning $l_{1}$. We call an element of $l_{1}$ positive if it is nonzero and has all the coordinates nonnegative. 
Corollary 1. Let $X$ be a set of positive elements of $l_{1}$ and suppose $f$ has the form

$$
f(x, \omega)=\Phi(\omega) x+H(x) G(x, \omega)
$$

with a random variable $\Phi: \Omega \rightarrow(0, \infty)$, a Borel $H: X \rightarrow \mathbb{R}$ and a randomvalued function $G: X \times \Omega \rightarrow X$. Assume that there exist a positive random variable $M$ and constants $\alpha>0, \beta \in(0,1)$ such that

$$
\begin{gathered}
E(M / \Phi)<\infty, \quad\|G(x, \omega)\| \leq M(\omega) \quad \text { for } x \in X, \omega \in \Omega, \\
|H(x)| \leq\|x\|^{1+\alpha}, \quad E\|f(x, \cdot)\|^{\alpha} \leq \beta\|x\|^{\alpha} \quad \text { for } x \in X .
\end{gathered}
$$

Then for every $x \in X$ the sequence

$$
\left(\frac{f^{n}(x, \omega)}{\prod_{k=1}^{n} \Phi\left(\omega_{k}\right)}\right)
$$

converges a.s. to a measurable function with positive values in $l_{1}$. Moreover, if $E|\log \Phi|<\infty$, then, for $x \in X$,

$$
\lim _{n \rightarrow \infty} \sqrt[n]{\left\|f^{n}(x, \cdot)\right\|}=\exp \{E \log \Phi\} \quad \text { a.s. }
$$

Proof. Fix $x \in X, x^{*} \in l_{1}^{*}$ and let $x_{1}^{*}, x_{2}^{*} \in l_{1}^{*}$ be positive functionals such that $x^{*}=x_{1}^{*}-x_{2}^{*}$. Replacing (if necessary) $x_{i}^{*}$ by $x_{i}^{*}+x_{0}^{*}$, where $x_{0}^{*}(x)=\sum_{n=1}^{\infty} x_{n}$, we may assume that

$$
|H(x)| \leq x_{i}^{*} x\|x\|^{\alpha} \quad \text { and } \quad x_{i}^{*} x>0 \quad \text { for } x \in X, i=1,2 .
$$

By Theorem 1, $\left(x_{i}^{*} \frac{f^{n}(x, \omega)}{\prod_{k=1}^{n} \Phi\left(\omega_{k}\right)}\right)$ converges a.s., hence so does $\left(x^{*} \frac{f^{n}(x, \omega)}{\prod_{k=1}^{n} \Phi\left(\omega_{k}\right)}\right)$, and the exceptional set is independent of $x^{*}$. Since $l_{1}$ is weakly sequentially complete, this shows that for a.e. $\omega \in \Omega^{\infty}$ the sequence

$$
\left(\frac{f^{n}(x, \omega)}{\prod_{k=1}^{n} \Phi\left(\omega_{k}\right)}\right)
$$

weakly converges and by the Banach-Schur property of $l_{1}$ (which means that every weakly convergent sequence is strongly convergent; see, e.g., [1; p. 200]) it converges in norm. Clearly, the limit is nonnegative and by the first part of (6) it is nonzero.

Note that Corollary 1 also holds if $X$ is a subset of an arbitrary finitedimensional normed space.

Now we proceed to the case where $\Phi=0$. The following simple lemma will be used in the proof of Theorem 3 .

LEMMA 1. If $u_{n}, R_{n}, v_{n}$ are integrable random variables such that

$$
u_{n} \leq R_{n} \leq v_{n}, \quad \sum_{n=1}^{\infty} E\left(v_{n}-u_{n}\right)<\infty
$$

and one of the series $\sum_{n=1}^{\infty} u_{n}, \sum_{n=1}^{\infty} v_{n}$ converges a.s., then so does $\sum_{n=1}^{\infty} R_{n}$. 
TheOREM 3. Assume that there exist positive random variables $\xi_{n}, \zeta_{n}$ on $\Omega_{n}$ and a constant $\gamma>1$ such that

$$
\|x\|^{\gamma} \xi_{n}(\omega) \leq\left\|\varphi_{n}(x, \omega)\right\| \leq\|x\|^{\gamma} \zeta_{n}(\omega) \quad \text { for } x \in X, \omega \in \Omega_{n}, n \in \mathbb{N} .
$$

If the series

$$
\sum_{n=1}^{\infty} \gamma^{-n} E \log \xi_{n}, \quad \sum_{n=1}^{\infty} \gamma^{-n} E \log \zeta_{n}
$$

converge and

$$
\sum_{n=1}^{\infty} \gamma^{-2 n} D^{2} \log \xi_{n}<\infty \quad \text { or } \quad \sum_{n=1}^{\infty} \gamma^{-2 n} D^{2} \log \zeta_{n}<\infty
$$

then for every $x \in X \backslash\{0\}$ the sequence

$$
\left(\left\|f^{n}(x, \cdot)\right\|^{\gamma^{-n}}\right)
$$

converges a.s. to a positive random variable.

Proof. Let

$$
F_{n}(x, \omega)=\frac{\left\|\varphi_{n}(x, \omega)\right\|}{\|x\|^{\gamma}}
$$

for $x \in X \backslash\{0\}, \omega \in \Omega_{n}, n \in \mathbb{N}$. Clearly

$$
F_{n}(x, \omega)>0 \quad \text { for } x \in X \backslash\{0\}, \omega \in \Omega_{n}, n \in \mathbb{N},
$$

and an easy induction shows that

$$
\left\|f^{n}(x, \omega)\right\|=\|x\|^{\gamma^{n}} \prod_{k=1}^{n} F_{k}\left(f^{k-1}(x, \omega), \omega_{k}\right)^{\gamma^{n-k}} .
$$

Therefore

$$
\log \left\|f^{n}(x, \omega)\right\|^{\gamma^{-n}}=\log \|x\|+\sum_{k=1}^{n} \gamma^{-k} \log F_{k}\left(f^{k-1}(x, \omega), \omega_{k}\right)
$$

and to get the a.s. convergence of (11) to a positive random variable it is enough to show that the series

$$
\sum_{k=1}^{n} \gamma^{-k} \log F_{k}\left(f^{k-1}(x, \omega), \omega_{k}\right)
$$

converges a.s. To this end put

$u_{n}(\omega)=\frac{\log \xi_{n}\left(\omega_{n}\right)}{\gamma^{n}}, R_{n}(\omega)=\frac{\log F_{n}\left(f^{n-1}(x, \omega), \omega_{n}\right)}{\gamma^{n}}, v_{n}(\omega)=\frac{\log \zeta_{n}\left(\omega_{n}\right)}{\gamma^{n}}$,

observe that (9) holds and assume that the first series in (10) converges. Since the $u_{n}$ are independent random variables and the series $\sum_{n=1}^{\infty} E u_{n}$ and $\sum_{n=1}^{\infty} D^{2} u_{n}$ converge, it follows that $\sum_{n=1}^{\infty} u_{n}$ converges a.s. Applying Lemma 1 we get the desired a.s. convergence of (12). 
Note that if $\xi_{n}$ and $\zeta_{n}$ do not depend on $n$, and $\log \xi_{1}$ and $\log \zeta_{1}$ are integrable, then the series $\sum_{n=1}^{\infty} u_{n}$ and $\sum_{n=1}^{\infty} v_{n}$ (defined in the above proof) converge absolutely a.s. This leads us to the following corollary.

Corollary 2. Assume that there exist positive random variables $\xi, \zeta$ on $\Omega$ and a constant $\gamma>1$ such that

$$
\|x\|^{\gamma} \xi(\omega) \leq\|f(x, \omega)\| \leq\|x\|^{\gamma} \zeta(\omega) \quad \text { for } x \in X, \omega \in \Omega .
$$

If $\log \xi$ and $\log \zeta$ are integrable then for every $x \in X \backslash\{0\}$ the sequence

$$
\left(\left\|f^{n}(x, \cdot)\right\|^{\gamma^{-n}}\right)
$$

converges a.s. to a positive random variable.

Acknowledgments. This research was supported by the Silesian University Mathematics Department (Iterative Functional Equations and Real Analysis program).

\section{References}

[1] C. D. Aliprantis and O. Burkinshaw, Positive Operators, Academic Press, Orlando, FL, 1985.

[2] K. Baron, On the convergence of sequences on iterates of random-valued functions, Aequationes Math. 32 (1987), 240-251.

[3] K. Baron and W. Jarczyk, Random-valued functions and iterative functional equations, ibid. 67 (2004), 140-153.

[4] K. Baron and M. Kuczma, Iteration of random-valued functions on the unit interval, Colloq. Math. 37 (1977), 263-269.

[5] Ph. Diamond, A stochastic functional equation, Aequationes Math. 15 (1977), 225233.

[6] R. Kapica, Sequences of iterates of random-valued vector functions and continuous solutions of a linear functional equation of infinite order, Bull. Polish Acad. Sci. Math. 50 (2002), 447-455.

[7] -, Convergence of sequences of iterates of random-valued vector functions, Colloq. Math. 97 (2003), 1-6.

[8] - , Theorems of Thron's type for random-valued vector functions and the KreinRutman theorem, Ann. Polon. Math. 85 (2005), 13-24.

[9] M. G. KreĬn and M. A. Rutman, Linear operators leaving invariant a cone in a Banach space, Uspekhi Mat. Nauk (N.S.) 3 (1948), no. 1, 3-95 (in Russian); English transl.: Functional Analysis and Measure Theory, Amer. Math. Soc. Transl. 10, 1962, 199-325.

[10] M. Kuczma, Normalizing factors for iterates of random-valued functions, Prace Naukowe Uniwersytetu Śląskiego w Katowicach, Prace Mat. 6 (1975), 67-71.

[11] M. Kuczma, B. Choczewski and R. Ger, Iterative Functional Equations, Encyclopedia Math. Appl. 32, Cambridge Univ. Press, 1990.

[12] W. J. Thron, Sequences generated by iteration, Trans. Amer. Math. Soc. 96 (1960), $38-53$. 
[13] J. Walorski, Convex solutions of the Schröder equation in Banach spaces, Proc. Amer. Math. Soc. 125 (1997), 153-158.

[14] —, Thron's theorems in Banach spaces, Grazer Math. Ber. 334 (1997), 233-240.

Institute of Mathematics

Silesian University

Bankowa 14

40-007 Katowice, Poland

E-mail: rkapica@ux2.math.us.edu.pl

Received 18.2.2005

and in final form 30.11.2006 